\title{
Determinantes de la Productividad Laboral para las Empresas Ecuatorianas en el Periodo 2009-2014
}

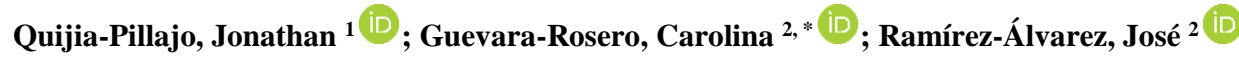 \\ ${ }^{1}$ Ingeniería en Ciencias Económicas y Financieras, Escuela Politécnica Nacional, Quito, Ecuador \\ ${ }^{2}$ Departamento de Economía Cuantitativa, Escuela Politécnica Nacional, Quito, Ecuador
}

\begin{abstract}
Resumen: Este estudio determina los factores que afectan la productividad laboral de las empresas en Ecuador, incorporando factores externos a la empresa como las economías de aglomeración. Utilizando la información a nivel de empresas de la encuesta de ciencia, tecnología e innovación de los años 2011 y 2014, se emplea un modelo de regresión múltiple y se estima la productividad a través del método de mínimos cuadrados ordinarios. Los resultados indican que un aumento de un punto porcentual de la proporción de trabajadores con educación terciaria aumenta la productividad laboral en $1 \%$. Si las empresas exportan o se adhieren a un grupo empresarial, su productividad laboral aumentaría en $41 \%$ y $27,7 \%$, respectivamente. Otros factores que aumentan la productividad son el estatus multiplanta y la inversión de capital fijo. Además de los factores individuales, los factores externos como la densidad de empresas y la competencia en un sector y región dados pueden incentivar la productividad individual de las empresas.
\end{abstract}

Palabras clave: Empresas, Productividad laboral, Ecuador.

\section{Determinants of Labor Productivity for Ecuadorian Companies in the Period 2009-2014}

\begin{abstract}
This study determines the factors that affect the labor productivity of firms in Ecuador, introducing for the first time external factors such as agglomeration economies. Using the information at the firm level of the Science, Technology and Innovation Survey of 2011 and 2014, a multiple regression model is estimated with the Ordinary Least Squares method. The results indicate that an increase of one percentage point in the proportion of workers with tertiary education leads to an increase of labor productivity of $1 \%$. If firms export or operate in a corporative group, their productivity would increase in $41 \%$ and $27,7 \%$, respectively. Other factors that increase the productivity are the multiplant status and the investment in fixed capital Apart from these individual factors, the external factors such as the density and the level of competition of the firms in a given sector and region boost the individual productivity of firms.
\end{abstract}

Keywords: Firms, labor productivity, Ecuador.

\section{INTRODUCCIÓN}

La productividad es uno de los factores más importantes en el crecimiento económico. A nivel micro, la productividad determina la competitividad de las empresas para permanecer activas en el mercado (Medina, 2010), y a nivel macro, la productividad constituye un elemento clave para el crecimiento económico, la evolución de los salarios y el mantenimiento y mejora del nivel de vida de la población (Carro \& Gonzales, 2012).

Las diferencias de productividad son una de las principales fuentes de divergencia de ingresos entre países. Para disminuir la brecha existente, es entonces pertinente conocer los factores que impulsan la productividad en los países en vías de desarrollo. Este estudio contribuye a la literatura en este respecto, analizando el caso de Ecuador.

Según el Euromonitor International, para el año 2013, Ecuador tuvo una productividad laboral de apenas \$ USD 15370 , mientras que el promedio para América latina fue de \$ USD 18 351, y el promedio para los países de la OECD fue de \$ USD 77 929. Pese a que en el Plan Nacional de Desarrollo 2017-2021, el objetivo 5 establece que el Estado debe impulsar la productividad y competitividad para el crecimiento económico sostenible de manera redistributiva y solidaria, se evidencia que los niveles de la productividad aún son relativamente bajos, en relación con países desarrollados y con el contexto sudamericano.

*carolina.guevara@epn.edu.ec

Recibido: 20/02/2020

Aceptado: 06/01/2021

Publicado: 28/02/2021

10.33333/rp.vol47n1.02

CC BY 4.0 
En este sentido, el presente trabajo se enfoca en determinar los factores que afectan la productividad laboral a nivel de empresas. Para ello, se utiliza la encuesta de Ciencia, Tecnología e Innovación realizada por el Instituto Nacional Ecuatoriano de Censos para el año 2011 y 2014. Se emplea la metodología de Mínimos Cuadrados Ordinarios (MCO) cuyos resultados se los interpreta de manera correlacional.

Los principales resultados indican que, si una empresa contrata personal con altos niveles de educación, podría incrementar la productividad en un $1 \%$. El aumento del $1 \%$ en la inversión en capital fijo produce un incremento de la productividad del $2,24 \%$. Operar en los mercados internacionales y tener una empresa multiplanta incrementan la productividad en un $41 \%$ y $10,6 \%$, respectivamente. Adicionalmente, la adherencia a un grupo empresarial y el capital extranjero producen un incremento de la productividad del $27,7 \%$ y $33,3 \%$ respectivamente.

Además, se encontró que las empresas ecuatorianas no se benefician de la especialización. Lo que sí afecta positivamente a la productividad es la competencia y la densidad. Por un lado, la competencia impulsa a las empresas a adoptar nuevos procesos de producción más eficientes, para permanecer en el mercado. Por otro lado, la densidad favorece los mecanismos de aprendizaje, cooperación e intercambio que influyen positivamente en la productividad.

El presente trabajo se estructura de la siguiente manera: en la sección 2 se hace una revisión de la literatura concerniente a la productividad. La sección 3 muestra la metodología y estadísticas descriptivas básicas de los datos a utilizar: se presenta la fuente de datos, se expone la técnica econométrica a utilizarse y se describen las variables de análisis. A continuación, en la sección 4 se muestran los resultados de las variables que afectan a la productividad. Finalmente, en la sección 5 se presentan las conclusiones de la investigación, y se hace algunas recomendaciones de política.

\section{REVISIÓN DE LA LITERATURA}

Uno de los conceptos más relevantes en el análisis de los procesos de desarrollo económico en la actualidad es la productividad, ya que es uno de los factores más importantes para el crecimiento económico (López, Barcenilla, \& Mancebon, 2008). A nivel microeconómico, la alta productividad laboral implica altos ingresos reales tanto para el trabajador como para las empresas. Las empresas reducen sus costos y pueden tener una mejor posición en el mercado internacional (Medina, 2010).

En términos generales, la productividad es una característica empresarial que indica cuan eficiente es el uso de los recursos en la producción de bienes y servicios (Unger, Flores, \& Ibarra, 2014). Dicha característica depende no solo del trabajo y capital, sino también de la internalización de las empresas en los mercados internacionales, la composición del capital, de la política y de la geografía. A continuación, se realiza una breve revisión de esta literatura.

\section{1 Tamaño}

Benavente (2005) sugiere que las empresas grandes tienen mayor acceso a recursos tecnológicos, financieros, capital humano, lo cual las hace más competitivas y más productivas. Además, las empresas de mayor tamaño pueden ser más productivas por la presencia de economías de escala (Bogetic \& Olusi, 2013).

Por su parte, Chudnovsky, López \& Pupato (2006), en su trabajo acerca de la innovación y productividad para Argentina, encuentran una relación negativa y significativa con el tamaño de las empresas, es decir, las pequeñas empresas son más productivas que las grandes empresas. Los autores sugieren que las empresas pequeñas tienen la capacidad de adaptarse a cambios en el mercado, lo cual es mucho más difícil para las grandes empresas que tienen por lo general un gran número de empleados y gran capital invertido.

\subsection{Edad}

Según la literatura, el efecto de la edad de las empresas sobre su productividad es ambiguo. Por un lado, en los trabajos de Bogetic \& Olusi (2013) y Hall, Mairesse, \& Lotti (2012), se encuentra que las empresas más jóvenes tienden a ser más eficientes que las antiguas, debido a que, para las empresas antiguas, el conocimiento, las habilidades y destrezas se vuelven obsoletos e inducen el deterioro de la organización. En cambio, Roper, Du, \& Love (2008), en su estudio sobre la cadena de valor para China, determinan que a medida que los años de una empresa aumentan, las empresas son más productivas. El autor argumenta que las empresas con mayor tiempo en el mercado pueden acumular experiencia y conocimiento productivos, haciéndolas más eficientes, y por tanto más productivas.

\subsection{Multiplanta}

Crowley \& Mccann (2015) sugieren que las empresas que descentralizan su sistema de producción pueden aumentar su eficiencia con un proceso de producción dividido en fases, en establecimientos separados $\mathrm{y}$, en lugares favorables. La localización de estas unidades afecta tanto a la eficiencia como a la competitividad de las empresas, pues esto no solo está relacionado con el área de operaciones, sino también con la función comercial y financiera (Rosales, 2018).

\subsection{Estatus exportador}

La liberación y la intensificación del comercio desempeñan un papel importante sobre la productividad, a través de cinco canales potenciales: mayor variedad de los bienes intermedios, mejor calidad, difusión del conocimiento, amplificación de los efectos de aprendizaje y aumento del tamaño del mercado (Echavarría, Arbeláez \& Rosales, 2006; Álvarez \& Gutiérrez, 2011). El entorno competitivo se vuelve más fuerte cuando se opera en los mercados internacionales, por tanto, las empresas necesitan mejorar su eficiencia a un ritmo mucho más rápido, a diferencia de las empresas que compiten en el mercado nacional (Silva \& Africano, 2013). 


\section{5 Grupo empresarial}

Los grupos empresariales pueden facilitar aquellos intercambios que, de otro modo, no podrían ocurrir a través de los mercados. Por lo tanto, desempeñan un papel positivo en el desarrollo y rendimiento de la empresa, reduciendo los costos de transacción (Khanna \& Yafeh, 2007). Esto permite a las empresas dentro de los grupos emprender actividades económicas que no pueden ser mediadas a través de mercado.

Los grupos también pueden desempeñar un papel importante en la adquisición de tecnología y transferencia de conocimientos (Amsden \& Hikino, 1994). Como tal, la afiliación a grupos empresariales ha sido vista por algunos especialistas como una causa clave del rápido crecimiento de la productividad en el proceso dinámico de la industrialización.

\subsection{Capital extranjero}

Alderete \& Gutiérrez (2012), en su estudio sobre la productividad para las empresas colombianas, determinaron que el capital extranjero tiene un impacto positivo sobre la productividad laboral. Las empresas con capital extranjero tienen más acceso a las nuevas tecnologías por la relación con su casa matriz, la cual les ofrece asesoría técnica y otros servicios tecnológicos (Chong-Sup, 1997).

\subsection{Capital humano}

Las empresas que poseen personal con un alto nivel de estudios son más productivas (Álvarez \& Gutiérrez, 2011). Los empleados con altos niveles de educación poseen habilidades, conocimientos y capacidades que ayudan a las empresas a alcanzar objetivos y soluciones de manera eficiente (Yang, Lin \& Ma, 2010; Yunus, Said \& Law, 2014). Además, los trabajadores con altos niveles de educación poseen conocimientos que les permite ser capaces de aplicar, desarrollar y conservar tecnologías más sofisticadas (Alderete \& Gutiérrez, 2012).

\subsection{Sector industrial}

El sector donde operan las empresas también es un factor importante en la productividad. Los sectores difieren sustancialmente debido a la existencia de características particulares en cuanto a las oportunidades tecnológicas, las bases de conocimiento, y acumulación del avance técnico (Crowley \& Mccann, 2015; Echavarría, Arbeláez, \& Rosales, 2006; Željko \& Olasupo, 2013).

\subsection{Capital fijo}

La inversión destinada a los procesos de producción es clave para la productividad de las empresas (Benavente, 2005). Faruq \& Telaroli (2011) establecen que una mayor disponibilidad de capital físico en el proceso de producción permite a cada trabajador producir más en un periodo de tiempo determinado y, por lo tanto, ser más productivo.

\subsection{Maquinaria y equipo}

Los beneficios de la adquisición en maquinaria y equipo se pueden traducir en ahorros en la mano de obra, eficiencia de costos de operación y reducción de tiempos en los procesos de producción, provocando un incremento de la productividad (Ludym, Luzardo \& Rojas, 2018; Álvarez \& Gutiérrez, 2011).

\subsection{Economías de aglomeración, competencia y densidad}

Las economías de densidad, que consisten en la concentración geográfica de empresas de cualquier tipo, generan una serie de ventajas, tal como una gran disponibilidad de fuerza laboral, infraestructura y bienes públicos (Harrison, Kelley, \& Gant, 1996). Dichas ventajas pueden afectar positivamente a la productividad (Combes \& Gobillon, 2015; Ciccone \& Hall, 1996; Abel, Dey \& Gabe, 2011). En general, se encuentra que la proximidad geográfica entre las empresas y los empleados facilita los micro-mecanismos de cooperación, intercambio y aprendizaje (Duranton \& Puga, 2003).

Las economías de aglomeración se pueden distinguir por la estructura de la concentración de las empresas: dentro de una misma industria, también llamadas de especialización, o de diferentes industrias, también llamadas de diversificación. Estas economías de aglomeración desempeñan un rol importante en la productividad, a través de externalidades de conocimiento generadas (Guevara et al., 2018; Torrez \& Ordoñez, 2019). Según Glaeser et al. (1992), las economías de especialización o externalidades MAR permiten a las empresas acceder fácilmente a la mano de obra e insumos especializados que se requieren en la misma industria, con lo que pueden reducir costos y mejorar la calidad del producto (Baldwin, Brown, \& Rigby, 2008). La concentración espacial de empresas de la misma industria también puede facilitar los contactos cara a cara, el flujo de ideas entre las empresas de una industria y oportunidades de cooperar. Como consecuencia, el conocimiento y los métodos de producción se difunden y pueden surgir innovaciones que aumentan la productividad.

Las economías de diversificación o externalidades de Jacobs consisten en efectos positivos derivados de la concentración geográfica de empresas de diferentes industrias. De acuerdo con Jacobs (1969), dicha diversidad concentrada promueve la difusión del conocimiento, así como la disponibilidad de un gran panel de servicios que facilita la producción y da como resultado actividades innovadoras.

La intensidad de la competencia es otro determinante de la productividad, ya que la competencia local lleva a las empresas a innovar o adoptar nuevos procesos de producción más eficientes para mantenerse en el mercado (Porter, 1990; Porter, 1980).

\section{DATOS Y METODOLOGÍA}

\subsection{Datos}

Para la elaboración del presente trabajo, se emplea la información de la Encuesta Nacional de Actividades de Ciencia, Tecnología e Innovación (ACTI) realizada por el 
Instituto Nacional de Estadística y Censo (INEC), en los años 2011 y 2014. Esta encuesta tiene una cobertura nacional y cuenta con 7055 empresas para al año 2014 y 2815 para el año 2 011. En base a estos datos, se construyó un pool de datos a fin de obtener la mayor cantidad de información. Después de realizar la depuración de datos, que consistió en eliminar datos perdidos e identificar inconsistencias, la base final cuenta con 7957 observaciones.

\subsection{Descripción de las variables}

En la Tabla 1 se muestra la descripción de la variable independiente y de las variables independientes con su efecto esperado según la literatura revisada en la sección 2.

Tabla 1. Descripción de las variables

\begin{tabular}{|c|c|c|}
\hline Variables & Descripción & $\begin{array}{c}\text { Efecto } \\
\text { esperado }\end{array}$ \\
\hline \multicolumn{3}{|l|}{ Variable dependiente } \\
\hline Productividad laboral & \multicolumn{2}{|c|}{$\begin{array}{l}\text { Logaritmo de las ventas sobre el número de } \\
\text { empleados de la empresa i en el año t }\end{array}$} \\
\hline \multicolumn{3}{|l|}{$\begin{array}{l}\text { Variables } \\
\text { independientes }\end{array}$} \\
\hline Capital humano & $\begin{array}{l}\text { Porcentaje de empleados con } \\
\text { educación terciaria sobre el total de } \\
\text { empleados en la empresa i. }\end{array}$ & + \\
\hline Inversión en capital fijo & $\begin{array}{l}\text { Logaritmo de la inversión en capital } \\
\text { fijo de la empresa i en el año t }\end{array}$ & + \\
\hline Edad & $\begin{array}{l}\text { Tiempo que la empresa i lleva } \\
\text { operando hasta el año t. }\end{array}$ & $+/-$ \\
\hline Multiplanta & $\begin{array}{l}\text { Toma el valor de } 1 \text {, si la empresa i } \\
\text { tiene más de un establecimiento, y } 0 \\
\text { en caso contrario }\end{array}$ & + \\
\hline Estatus exportador & $\begin{array}{l}\text { Toma el valor de } 1 \text {, si la empresa i ha } \\
\text { realizado exportaciones, y } 0 \text { en caso } \\
\text { contrario. }\end{array}$ & + \\
\hline Capital extranjero & $\begin{array}{l}\text { Toma el valor de } 1 \text {, si el capital } \\
\text { extranjero de la empresa i es mayor } \\
\text { al } 10 \% \text {, y } 0 \text { en caso contrario. }\end{array}$ & + \\
\hline Grupo empresarial & $\begin{array}{l}\text { Toma el valor de } 1 \text {, si la empresa i } \\
\text { pertenece a un grupo empresarial, y } \\
0 \text { en caso contrario. }\end{array}$ & + \\
\hline $\begin{array}{l}\text { Gasto en maquinaria y } \\
\text { equipo }\end{array}$ & $\begin{array}{l}\text { Toma el valor de } 1 \text {, si la empresa i ha } \\
\text { realizado inversión en maquinaria y } \\
\text { equipo en el año t, y } 0 \text { en caso } \\
\text { contrario. }\end{array}$ & + \\
\hline \multicolumn{3}{|l|}{ Sectores industriales } \\
\hline Servicios & $\begin{array}{l}\text { Variable categórica que toma el } \\
\text { valor de } 1 \text { si la empresa i pertenece a } \\
\text { una de las categorías: manufactura, } \\
\text { comercio, minas y canteras y } \\
\text { servicios. La categoría de referencia } \\
\text { es Servicios. }\end{array}$ & $+/-$ \\
\hline T14 & $\begin{array}{l}\text { Toma el valor de } 1 \text { si la empresa } \\
\text { pertenece al año } 2014,0 \text { si pertenece } \\
\text { al año } 2011\end{array}$ & $+/-$ \\
\hline
\end{tabular}

Elaboración: Autor

Otras variables que están relacionadas con la productividad laboral son los índices de economías de aglomeración. A continuación de detalla la forma de cálculo de cada índice según Combes (2000).

Índice de especialización

$$
s p e_{s,}=\frac{e m p l_{s, p} / e m p l_{p}}{e m p l_{s} / e m p l}
$$

Donde empl $l_{s, p}$ es el número de empleados en el sector económico $\mathrm{s}$ en la provincia $\mathrm{p}$, empl $_{p}$ es empleo en la provincia $p, e m p l_{s}$ es el empleo del sector económico $s$ y empl es el empleo total.

\section{Índice de diversidad}

Si bien las economías de urbanización se miden comúnmente a través del inverso del índice Herfindahl, construido a partir de la participación de las industrias en el empleo local, con la excepción de la industria que se considera, esta variable se normaliza por la misma variable a nivel de país:

$$
d i v_{s, p}=\frac{1 / \sum_{S^{\prime}=1, s^{\prime} \neq s}^{S}\left(e m p l_{s^{\prime}, p} /\left(e m p l_{p}-e m p l_{s, p}\right)\right)^{2}}{1 / \sum_{S^{\prime}=1, s^{\prime} \neq s}^{S}\left(e m p l_{s^{\prime}} /\left(e m p l-e m p l_{s}\right)\right)^{2}}
$$

Donde $e m p l_{s^{\prime}, p}$ es el empleo del sector $s$ (sujeto de análisis) de la provincia $\mathrm{p}$, empl $_{p}$ es el empleo de la provincia $p$, $e m p l_{s, p}$ es el empleo del sector $s$ de la provincia $p, e m p l_{s^{\prime}}$ es el empleo del sector $s$ (sujeto de análisis), empl es el empleo total y empl $l_{s}$ es el empleo en el sector $s$. El numerador es máximo cuando todos los sectores, excepto el sujeto del análisis $s^{\prime}$, son del mismo tamaño en las ciudades. El índice mide el nivel de diversidad del sector económico s en la provincia p. Por lo tanto, no está necesariamente relacionado con el nivel de especialización de la industria que se analiza.

Índice de Competencia

$$
\operatorname{comp}_{s, p}=\frac{1 / \sum_{i}^{N}\left(e m p l_{i, s, p} / e m p l_{, s, p}\right)^{2}}{1 / \sum_{i}^{N}\left(e m p l_{i, s} / e m p l_{, s}\right)^{2}}
$$

Donde $e m p l_{i, s, p}$ es el empleo de la empresa $i$ perteneciente al sector $s$ y ubicada en la provincia $p, e m p l_{i, s}$ es el empleo de la empresa $\mathrm{i}$ en el sector s a nivel nacional, empl, ${ }_{s, p}$ es el empleo en el sector $s$, en la provincia p, y empl, es el empleo en el sector $s$.

Índice de densidad

$$
\operatorname{den}_{s, p}=\frac{f_{s, p} / \text { área }}{f_{s} / \text { área }}
$$

Donde $f_{s, p}$ es el número de empresas en el sector s localizada en la provincia p y áre $a_{p}$ es el área de la provincia p. En el denominador, $f_{s}$ es el número de empresas del sector s y área es la superficie total del país.

\subsection{Estadísticos descriptivos}

La productividad laboral promedio de las empresas ecuatorianas es de $\$ 306$ 804,3. Es decir, cada empleado por empresa genera en promedio aproximadamente un ingreso de 300 mil dólares al año. Desagregando la productividad por tamaño de las empresas se tiene lo siguiente: Las empresas pequeñas y grandes son las que presentan mayores niveles productividad laboral. Las empresas pequeñas tienen una 
productividad de USD 276 141,7, mientras que las empresas grandes tienen una productividad de USD 776 071,2.

En cuanto a las variables independientes dicotómicas, se tiene que el $30,5 \%$ de las empresas poseen un capital humano con educación terciaria, el $29 \%$ son de carácter multiplanta y el $11 \%$ son de carácter exportador. Asimismo, se tiene que el $10 \%$ de las empresas poseen capital extranjero alto y el $16 \%$ de las empresas pertenecen a un grupo empresarial. Respecto a las variables continuas, las empresas ecuatorianas en promedio invierten alrededor de $\$ 922756,3$ anuales en capital fijo, y tienen una edad promedio de 18 años.

Tabla 2. Estadísticos descriptivos

\begin{tabular}{|c|c|c|c|c|}
\hline Variables & Media & Ds. & Mín. & Máx. \\
\hline \multicolumn{5}{|l|}{ Var. dependiente } \\
\hline Productividad laboral & 306804,3 & 3131568 & 1 & 152175700 \\
\hline \multicolumn{5}{|l|}{ Productividad por tamaño } \\
\hline Pequeña & 276141,7 & 2570568 & 11,33 & 133238300 \\
\hline Mediana & 166726,5 & 526439,4 & 88,25 & 10401800 \\
\hline Grande & 776071,2 & 6973437 & 1 & 152175700 \\
\hline Var. independientes & Media & Ds. & Mín. & Máx. \\
\hline Inv. capital fijo & 922756,3 & 18400000 & 1 & 1069394000 \\
\hline Edad & 18 & 14,2 & 1 & 196 \\
\hline SPECsp & 1,08 & 0,6 & 0,04 & 12,3 \\
\hline DIVERsp & 2,03 & 0,4 & 1 & 3 \\
\hline COMPsp & 0,26 & 0,1 & 0,01 & 0,6 \\
\hline DENsp & 4,38 & 3 & 2,96 & 10 \\
\hline Capital humano & $30,50 \%$ & & & \\
\hline Multiplanta & $29,20 \%$ & & & \\
\hline Estatus exportador & $11,20 \%$ & & & \\
\hline Capital extranjero & $9,60 \%$ & & & \\
\hline Grupo empresarial & $15,90 \%$ & & & \\
\hline Gasto en maquinaria & $27,20 \%$ & & & \\
\hline \multicolumn{5}{|l|}{ Sectores industriales } \\
\hline Comercio & $25,3 \%$ & & & \\
\hline Minas y canteras & $3,1 \%$ & & & \\
\hline Manufactura & $29,2 \%$ & & & \\
\hline \multicolumn{5}{|l|}{ Servicios } \\
\hline Transporte & $6.5 \%$ & & & \\
\hline Act. profesionales & $7.25 \%$ & & & \\
\hline Alojamiento & $5.57 \%$ & & & \\
\hline Otros servicios & $22.97 \%$ & & & \\
\hline
\end{tabular}

Fuente: Encuesta de actividades de Ciencia, Tecnología e Innovación 2011 y 2014 reportado por el INEC. Elaborado por: El autor.

Nota: El sector "Otros Servicios" comprenden: Sector financiero e inmobiliario, Comunicaciones, Salud, Agua Potable y Energía Eléctrica

En cuanto a los sectores industriales, se puede observar que la de industria de servicios es la más predominante, seguida de la industria manufactura. Estos resultados nos indican que la industria de servicios es un sector muy importante para el Ecuador, ya que podría generar empleo, inversión, entre otros. Finalmente, respecto a las economías de aglomeración, urbanización, competencia y densidad, se tiene que las provincias ecuatorianas tienen una especialización relativamente baja (media 1,08), son altamente diversas (media 2,03), y presentan una baja dispersión del empleo (media 0,26). Además, se puede establecer que existen alrededor de cuatro empresas por kilómetro cuadrado en un sector s y una provincia p. Analizando un poco más detalladamente la densidad en las provincias se tiene lo siguiente en la Tabla 3. La provincia más densa es la provincia de Pichincha, con alrededor de 8 empresas por kilómetro cuadrado. En segundo y tercer lugar se tiene a las provincias de Tungurahua y el Guayas con 5 empresas y 4 empresas por kilómetro cuadrado, respectivamente. Las provincias menos densas son las provincias de Pastaza y Morona Santiago con un valor de 0,4 y 0,7 respectivamente. Estas provincias pertenecen a la región amazónica por lo que es muy difícil abrir una empresa, ya implicaría altos costos de transporte, falta de mano de obra especializada etc.

Tabla 3. Ranking de las provincias más densas

\begin{tabular}{|c|c|c|}
\hline Posición & Provincia & DENsp \\
\hline 1 & Pichincha & 8,16 \\
\hline 2 & Tungurahua & 4,95 \\
\hline 3 & Guayas & 4,05 \\
\hline 4 & El Oro & 3,16 \\
\hline 5 & Azuay & 3,08 \\
\hline 6 & Santo Domingo & 2,08 \\
\hline 7 & Imbabura & 1,51 \\
\hline 8 & Santa Elena & 1,01 \\
\hline 9 & Cotopaxi & 0,87 \\
\hline 10 & Chimborazo & 0,84 \\
\hline 11 & Los Ríos & 0,77 \\
\hline 12 & Carchi & 0,70 \\
\hline 13 & Manabí & 0,66 \\
\hline 14 & Loja & 0,66 \\
\hline 15 & Cañar & 0,63 \\
\hline 16 & Bolívar & 0,38 \\
\hline 17 & Esmeraldas & 0,24 \\
\hline 18 & Zamora Chinchipe & 0,22 \\
\hline 19 & Galápagos & 0,21 \\
\hline 20 & Sucumbíos & 0,14 \\
\hline 21 & Orellana & 0,13 \\
\hline 22 & Napo & 0,08 \\
\hline 23 & Morona Santiago & 0,07 \\
\hline 24 & Pastaza & 0,04 \\
\hline
\end{tabular}

Elaboración: El autor

\subsection{Metodología}

Con el fin de hallar los factores determinantes de la productividad laboral considerando las variables independientes antes mencionadas, se utiliza un pool de datos que se estima a través del método de mínimos cuadrados ordinarios. Este método consiste en minimizar la suma de los cuadrados de las distancias entre los valores de los datos y los 
valores ajustados por el modelo. Formalmente, este modelo tiene la siguiente especificación:

$$
y_{i}=\alpha_{0}+\alpha x_{i}+\delta T+\epsilon_{i}
$$

donde $y_{i}$ el logaritmo natural de la productividad laboral, $\boldsymbol{x}_{i}$ es un vector de variables explicativas relacionadas con la productividad laboral, $\boldsymbol{\alpha}$ es el vector de parámetros, $T$ es una variable dicotómica relacionada con el año de la encuesta, $\delta$ es el coeficiente correspondiente a este año, y $\epsilon_{i}$ es el término de error que sigue una distribución normal.

Para la validación del modelo, se analiza la heterocedasticidad, multicolinealidad, la posibilidad de que exista una variable omitida y la normalidad en los residuos. Para analizar el posible error de especificación en la forma funcional, se utiliza el test de Ramsey. Los resultados nos indican que no se puede rechazar la hipótesis de que el modelo tiene una forma lineal (Prob $>\mathrm{F}=0,0000$ ). Respecto a la multicolinealidad, se utiliza el factor de inflación de la varianza (VIF). Los resultados nos indican que no hay un problema de multicolinealidad pues en promedio el VIF tiene un valor de 1,73, y de forma individual el VIF no es mayor a 3,74. Al analizar la distribución de los errores estandarizados, se identificaron valores atípicos que superan las dos desviaciones estándar. Estas observaciones (387) fueron eliminadas. Finalmente, para analizar la normalidad de los residuos, se utilizan los tests de Skewnes y Kurtosis, los cuales indican que no hay normalidad. Sin embargo, por el gran número de observaciones y el tratamiento adecuado de datos atípicos, los estimadores tienden a ser insesgados y consistentes. Además, los datos no se alejan de manera significativa de la curva de normalidad como se puede observar en la Figura 1. Es necesario advertir que la estimación del modelo no corrige la endogeneidad de las variables independientes (es decir, la posibilidad que la productividad afecte por ejemplo el tamaño de la empresa). En este sentido, los resultados que se muestran a continuación son correlacionales y no causales.

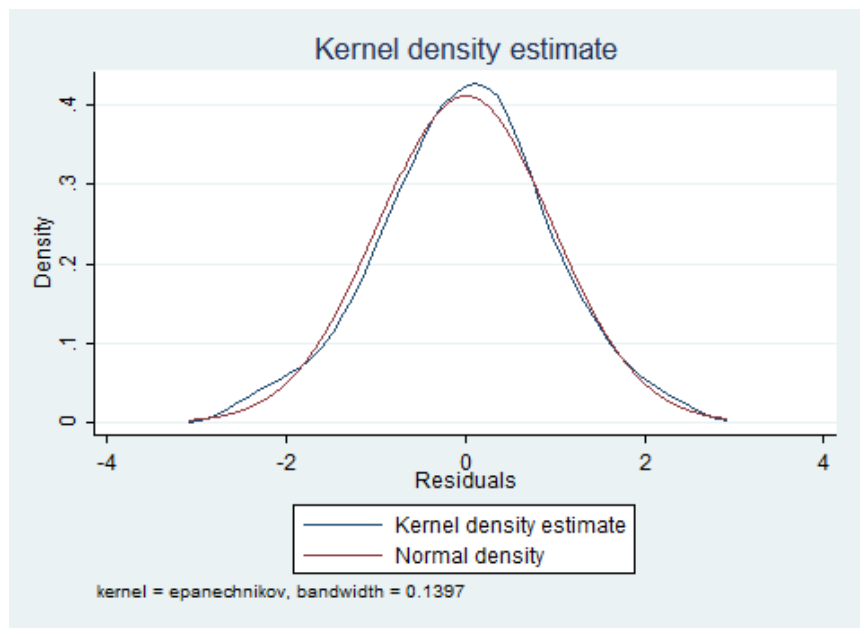

Figura 1. Prueba gráfica de normalidad de Kernel

\section{RESULTADOS}

En la Tabla 4 se presentan los resultados de la estimación de la productividad laboral para las empresas ecuatorianas.

Tabla 4. Estimación de la productividad laboral

\begin{tabular}{|c|c|c|}
\hline Variables independientes & $\begin{array}{c}\text { Productividad } \\
\text { laboral }\end{array}$ & $\begin{array}{c}\text { Error } \\
\text { estándar }\end{array}$ \\
\hline Capital humano & $1.006^{* * *}$ & $(0,05)$ \\
\hline Ln capital fijo & $0.0224^{* * *}$ & $(0,0022)$ \\
\hline Edad & $0.0053^{* * *}$ & $(0,0015)$ \\
\hline $\operatorname{Edad}^{\wedge} 2$ & -0.0000241 & $(0,00001)$ \\
\hline Multiplanta & $0.106^{* * *}$ & $(0,025)$ \\
\hline Exportador & $0.4102^{* * *}$ & $(0,039)$ \\
\hline Capital extranjero & $0.333^{* * *}$ & $(0,044)$ \\
\hline Grupo empresarial & $0.277^{* * *}$ & $(0,033)$ \\
\hline Maquinaria y Equipo & $-0.119^{* * * *}$ & $(0,027)$ \\
\hline $\mathrm{T} 14$ & $0.7648^{* * *}$ & $(0,029)$ \\
\hline \multicolumn{3}{|l|}{ Índices de aglomeración } \\
\hline Especialización & $-0.1025^{* * *}$ & $(0,023)$ \\
\hline Diversidad & 0.026 & $(0,035)$ \\
\hline Competencia & $0.4246^{* * *}$ & $(0,132)$ \\
\hline Densidad & $0.0279^{* * *}$ & $(0,0063)$ \\
\hline \multicolumn{3}{|c|}{ Sectores industriales (ref. Otros servicios) } \\
\hline Comercio & $1.166^{* * *}$ & $(0,035)$ \\
\hline Manufactura & $0.172^{* * *}$ & $(0,035)$ \\
\hline Minas y canteras & $-0.296^{* * *}$ & $(0,081)$ \\
\hline Transporte & -0.0148 & $(0,056)$ \\
\hline Actividades profesionales & $-0.210^{* * *}$ & $(0,049)$ \\
\hline Alojamiento & $-0.426^{* * *}$ & $(0,045)$ \\
\hline Constante & $9.08^{* * *}$ & $(0,088)$ \\
\hline$N$ & 7570 & \\
\hline $\mathbf{F}$ & $284,56(0,0000)$ & \\
\hline$R_{a j}^{2}$ & 0,4419 & \\
\hline \multicolumn{3}{|l|}{ Prueba de normalidad } \\
\hline Skewness & $\mathrm{p}$-valor $=0,0014$ & \\
\hline Kurtosis & $\mathrm{p}$-valor $=0,7634$ & \\
\hline Prueba Ramsey & $5,39(0,0011)$ & \\
\hline Mean VIF & 1,73 & \\
\hline
\end{tabular}

Nota: Variables con $* * *$ son significantes al $1 \%$, con $* *$ son significantes al $5 \%, \mathrm{y} *$ son significantes al $1 \%$. El error estándar está en el paréntesis. El sector de otros servicios es la categoría de referencia.

El modelo tiene un coeficiente de determinación ajustado de 0,44 , lo que significa que las variables independientes explican el $44 \%$ de la variabilidad de la productividad laboral. Analizando el estadístico F, se puede determinar que las variables en su conjunto explican a la productividad laboral, pues no se rechaza la hipótesis nula de que los coeficientes son diferentes de cero (Prob $>\mathrm{F}=0,0000$ ).

Una vez comprobado que el modelo tiene un buen nivel de ajuste, analizamos la significancia estadística y económica de las variables independientes. El capital humano tiene un impacto positivo y significativo sobre la productividad laboral 
de las empresas. Si las empresas aumentan la proporción de empleados con educación terciaria en un punto porcentual, entonces podrían incrementar su productividad en un $1 \%$. El recurso humano capacitado provee a las empresas de conocimiento y eficiencia para la ejecución del trabajo, generando así un incremento en el nivel de productividad (Álvarez et al., 2011; Yang et al. 2010; Yunus et al., 2014).

La inversión en capital fijo tiene un efecto positivo y significativo sobre la productividad laboral. Si esta inversión crece en $1 \%$, entonces la productividad de las empresas podría aumentar en 0,022\%. El aumento y las mejoras de capital se traducen en cambios en las prácticas, productos y mejores procedimientos de la empresa, lo cual genera un incremento en el nivel de productividad (Benavente J., 2005; Faruq et al., 2011). La edad de las empresas incide de manera positiva su productividad laboral al $99 \%$ de confianza. Se tiene que, por cada año de operación de la empresa, su productividad aumenta en $0,53 \%$. Además, se puede advertir que esta relación no tiene una forma de $\mathrm{U}$ invertida, pues el término al cuadrado no es significativo.

El estatus multiplanta tiene un efecto positivo y significativo sobre la productividad laboral de las empresas. Si una empresa es de carácter multiplanta, esta empresa podría incrementar su productividad en un $10,6 \%$. En efecto, las empresas que dividen sus procesos de producción en fases y las localizan en establecimientos separados ubicados en lugares favorables, mejoran su productividad ya que se vuelven más eficientes (Crowley \& Mccann, 2015; Rosales, 2018).

El estatus exportador tiene un efecto positivo y significativo sobre la productividad. Si una empresa e s exportadora, esta podría incrementar su productividad en un $41,02 \%$. Cuando las empresas operan en mercados internacionales pueden tener ventajas como: una mayor variedad de los bienes intermedios, mejor calidad, difusión del conocimiento, amplificación de los efectos de aprendizaje y aumento del tamaño del mercado (Echavarría, Arbeláez \& Rosales, 2006; Álvarez \& Gutiérrez, 2011).

Las variables grupo empresarial y capital extranjero, tienen un efecto positivo y significativo sobre la productividad de las empresas. Si las empresas cuentan con capital extranjero pueden incrementar su productividad en un 33,3\%, y si forman parte de un grupo empresarial pueden incrementar su productividad en un $27,7 \%$. Las empresas con capital extranjero pueden acceder a recursos tecnológicos por su relación con su casa matriz, mientras que las empresas que pertenecen a un grupo empresarial pueden emprender actividades económicas que no pueden ser mediadas a través del mercado, tal como actividades en finanzas, trabajo y productos (Aldarete et al., 2012; Chong-Sup, 1997; Khanna et al., 2005; Amsden et al., 1994).

El gasto en maquinaria y equipo tiene un efecto negativo en la productividad. Aquellas empresas que registran este tipo de gastos tienen una productividad $0,12 \%$ menor que aquellas que no lo tienen. A priori se esperaba un efecto positivo, ya que este tipo de inversión mejoraría la productividad. Una de las razones de este posible resultado es que existe una mayor proporción de empresas que pertenecen al sector de servicios, las cuales no tienen la necesidad de invertir grandes cantidades en este tipo de inversión (Maquinaria y equipo) sino en otros recursos especializados.

En cuanto a las actividades económicas, el sector comercio y manufactura son más productivas que el sector de servicios, en $116 \%$ y $17 \%$, respectivamente. Este hecho puede deberse a que dichos sectores son los que elaboran y comercializan mayor cantidad de productos, por lo que sus niveles de utilidad por trabajador serán mayores. Cuando desagregamos el sector de servicios, se puede comprobar que los servicios de alojamiento y actividades profesionales son menos productivos que el resto de servicios en la economía. Este hecho puede ser explicado por la alta rentabilidad que brinda el sector financiero y que compone gran parte del sector de referencia.

De acuerdo con la teoría, la especialización de los sectores industriales tiene un efecto positivo sobre la productividad mediante las externalidades MAR; sin embargo, para el caso ecuatoriano se muestra un efecto negativo. En otras palabras, la aglomeración de las empresas en una misma industria disminuye la productividad de las empresas. Guevara et al., (2018) y Torrez \& Ordoñez (2018) obtienen los mismos resultados y establecen que el efecto de las externalidades de la aglomeración depende del ciclo de vida de la industria. Al parecer, la industria ecuatoriana elabora productos estándar y maduros, para los cuales se mantiene un mismo nivel de productividad. Algunos factores que podrían estar impidiendo la generación de efectos positivos de especialización sobre la productividad son la baja calidad del transporte, infraestructura y bajos niveles de innovación.

La competencia tiene un impacto positivo y significativo sobre la productividad, por lo que un aumento en la competencia local de las empresas conduce a un mayor retorno productivo. La intensidad de la competencia es el determinante más fuerte para la productividad de las empresas, ya que la competencia local lleva a las empresas a innovar o adoptar nuevos procesos de producción más eficientes para mantenerse en el mercado (Porter, 1990; Porter, 1980).

Finalmente, la densidad de las empresas también tiene un efecto positivo y significativo sobre la productividad. Ante el aumento de 1 empresa por kilómetro cuadrado, la productividad puede incrementar en un $2,79 \%$. La proximidad geográfica entre las empresas y empleados favorece y facilita los mecanismos de cooperación, intercambio y aprendizaje (Combes \& Gobillon, 2015; Guevara et al. 2018; Ciccone \& Hall, 1996; Abel, Dey \& Gabe, 2011).

\section{CONCLUSIONES}

El análisis de los determinantes de la productividad en el Ecuador muestra que las características de las empresas que impulsan en mayor medida la productividad son: el capital humano capacitado, las relaciones con otras empresas a través de un grupo empresarial, la apertura comercial vía exportaciones y la inversión de capital extranjero. Este tipo de factores generan beneficios de productividad como mayor disponibilidad de bienes intermedios, acceso a nuevas tecnologías, asesoría técnica y difusión del conocimiento. 
La contribución a la literatura existente es que, además de variables internas a la empresa sobre las cuales ésta tiene control, se consideran factores externos como las economías de aglomeración. Entre los factores relacionados a este último aspecto, la competencia y la densidad de un sector económico impulsan la productividad de las empresas. Por un lado, la competencia obliga a las empresas a adoptar nuevos procesos de producción, para hacerlas más eficientes y permanecer en el mercado. Por otro lado, la densidad favorece los mecanismos de aprendizaje, cooperación e intercambio que influyen positivamente en la productividad. Pese a estos resultados, se encontró que la especialización de un sector tiene un efecto negativo sobre la productividad. Las empresas ecuatorianas no son capaces de absorber dicha externalidad por la estructura de las industrias basada en la producción de productos maduros y estándar.

Para impulsar la productividad de las empresas, en base a los resultados, se recomienda que las empresas se enfoquen en incorporar empleados con altos niveles de educación, pues este tipo de personal posee un dominio de conocimiento más amplio que influiría en la productividad. Además, se debe fomentar la interacción entre empresas en cualquiera de sus formas, ya sea a través de las exportaciones o alianzas en grupos empresariales, para generar nuevos conocimientos e innovación.

En términos contextuales, es recomendable impulsar un entorno de mayor competencia y densidad, puesto que, en este tipo de entorno, las empresas podrían aumentar los niveles de productividad.

Se propone como políticas públicas para estimular las productividad laboral de las empresas ecuatorianas: i) incentivar la formación del capital humano mediante programas de capacitación cofinanciados por el Estado en áreas claves del proceso de producción de la empresa, ii) promover la participación y cooperación de las compañías a través de proyectos de desarrollo conjunto que posean beneficios tributarios y acceso a bajas tasas de interés, y iii) establecer exenciones arancelarias mediante tratados comerciales que faciliten la apertura comercial,

Para finalizar, este estudio se puede extender utilizando la productividad total de factores en vez de la productividad laboral. La primera medida permite considerar la contribución del capital y la tecnología en la producción. En este estudio no se utilizó dicha medida por la limitación de disponibilidad de dicha información en la encuesta de innovación. Sin embargo, se podría recurrir al Censo Económico 2010 que cuenta con dicha información para un solo año. Otra línea de investigación futura es el enfoque en un grupo determinado de empresas, ya sea por sector o por tamaño, para llegar a identificar más específicamente la dinámica de productividad de ciertas empresas.

\section{REFERENCIAS}

Abel, J., Dey, I., \& Gabe, T. (2011). Productivity and the Density of Human Capital. Federal Reserve Bank of New York.

Alderete, M., \& Gutiérrez, L. (2012). TIC y productividad en las industrias de servicios en Colombia. Scielo, Lectura de Economì Medellin., 77: 163-188.

Álvarez, I., \& Gutiérrez, C. (2011). Factores determinantes de la productividad en la industria española de bienes de equipo. Instituto Complutense de estudios, Madrid.

Amsden, A., \& Hikino, T. (1994). Project execution capability, organizational know-how and conglomerate corporate growth in late industrialization. Industrial and Corporate Change, 3: 111-148.

Baldwin, J., Brown, M., \& Rigby, D. (2008). Agglomeration Economies: Microdata Panel Estimates from Canadian Manufacturing. Journal of Regional Science, 50.10.1111/j.1467-9787.2010.00675.x. .

Benavente, J. (2002). Determinants of Industrial Research and Innovation:The case of Chile. University of Oxford, 574.

Bogetic, Z., \& Olusi, O. (2013). Drivers of Firm-Level Productivity in Russia's Manufacturing Sector. Policy Research Working Paper No. 6572. Washington, DC: World Bank.

Carro, R., \& Gonzales, D. (2012). Productividad y competitividad . Universidad Nacional de Mar del Plata-Argentina .

Chong-Sup, K. (1997). Los efectos de la apertura comercial y de la inversión extranjera directa en la productividad del sector manufacturero mexicano. El Trimestre Económico, 64: 365-390.

Chudnovsky, D., López, A., \& Pupato, G. (2006). Innovation and productivity in developing countries in developing countries: A study of argentine manufacturing firmas 1992-2001. Research Policy, 35(2): 266-288. 10.1016/j.respol.2005.10.002

Ciccone, A., \& Hall, R. (1996). Productivity and the Density of Economic Activity. American Economic Review, 86(1): 54-70. 10.2307/2118255

Combes, P. P. (2000). Economic Structure and Local Growth: France, 1984-1993. Journal of Urban Economics, 47(3), 329-355.

Combes, P., \& Gobillon, L. (2015). The Empirics of Agglomeration Economies. Handbook of Urban and Regional Economics, 5: 247-348. 
Crowley, F., \& Mccann, P. (2015). Innovation and Productivity in Irish Firms. Spatial Economic Analysis, 10(2): 181-204.

Duranton, G., \& Puga, D. (2003). Micro-foundations of urban agglomeration economies. Handbook of Regional and Urban Economics, 48: 2063-2117.

Echavarría, J., Arbeláez, M., \& Rosales, M. (2006). La productividad y sus determinantes:El Caso de la Industria Colombiana. Revista Desarrollo y Sociedad, Universidad de los Andes., Disponible en: RePEc:col:000090:003064.

Faruq, H., \& Telaroli, P. (2011). Factors Affecting Manufacturing and Agricultural Productivity Trends among Asian Countries. ASEAN Economic Bulletin, 28(1): 45-60. 10.2307/41317191

Glaeser, E. K. (1992). Growth in cities. Journal of Political Economy, 100(6): 1126-1152.

Guevara, C., Riou, S., \& Bernard, C. (2019). Agglomeration externalities in Ecuador: do urbanization and tertiarization matter? Regional Studies, 53(5), 706719. DOI: $10.1080 / 00343404.2018 .1470325$

Guevara, C., S., R., \& Autant, C. (2015). Agglomeration externalities and urbanization in Ecuador. 55th of the European Regional Science Association, Lisbon, Portugal., https://halshs.archives-ouvertes.fr/halshs01212174

Hall, B., Lotti, F., \& Mairesse, J. (2009). Innovation and productivity in SMEs: empirical evidence for Italy. Small Business Economics, 33:1, 13-33. $10.1007 / \mathrm{s} 11187-009-9184-8$

Harrison, B., Kelley, M., \& Gant, J. (1996). . Specialization versus diversity in local economies: the implications for innovative private-sector behaviour. Journal of Policy Development and Research, 61-93.

Jacobs, J. (1969). The Economies of Cities. Random House. New York.

Khanna, T., \& Yafeh, Y. (2007). Business Groups in Emerging Markets: Paragons or Parasites? . Journal of Economic Literature, 45(2), 331-372. DOI: 10.1257/jel.45.2.331

López, C., Barcenilla, S., \& Mancebon, M. (2008). La productividad total de los factores en los países desarrollados. Componentes y factores determinantes. Ekonomias.

Ludym, J., Luzardo, M., \& Rojas, M. (2018). Factores Determinantes de la Productividad Laboral en Pequeñas y Medianas Empresas de Confecciones del Área Metropolitana de Bucaramanga, Colombia. Información Tecnológica, 29(5): 175-186.
http://dx.doi.org/10.4067/S0718-

07642018000500175

Medina, J. (2010). Modelo Integral de productividad, aspectos importantes para su implementación. Revista Escuela de Adimistración de Negocios, 69, 110-119.

Porter, M. (1990). The Competitive Advantage of Nations. Harvard Bussines Review.

Porter, M. (1998). The Competitive Advantage of Nations. Harvard Business Review.

Roper, S., Du, J., \& Love, J. (2008). Modelling the innovation value chain. Research Policy, 37: 961-977.

Rosales, F. (2018). Teoría de localización de una planta industrial. Universidad Mariano Galvez, Guatemala.

Silva, A., \& Africano, A. P. (2013). ¿Las empresas más productivas se autoseleccionan para exportar? Aplicación de una prueba para el caso de Portugal. Investigación Económica, 72(283), 135-161. 10.1016/S0185-1667(13)72589-X

Torrez, T., \& Ordoñez, J. (2019). Agglomeration economies and urban productivity. REGIÓN, 6: 17-24.

Unger, K., Flores, D., \& Ibarra, J. (2014). Productividad y capital humano. El trimestre económico, 909-941.

Yang, T. y. (2012). Using a Systematic Organization Change Approach to Improve Productivity and Application with a Case Study on Semiconductor Manufacturing Company. doi: 10.6220/joq.2012.19(1).03, 39-58.

Yunus, N., Said, R., \& Law, S. (2014). Do cost of training, education level and R\&D investment matter towards influencing labour productivity? Jurnal Ekonomi Malaysia, 48(1), 133-142. DOI: 10.17576/JEM2014-4801-11

Željko, B., \& Olasupo, O. (2013). Drivers of Firm-Level Productivity in Russia's Manufacturing Sector. World Bank Policy Research Paper Series No. 6572. 


\section{BIOGRAFÍAS}

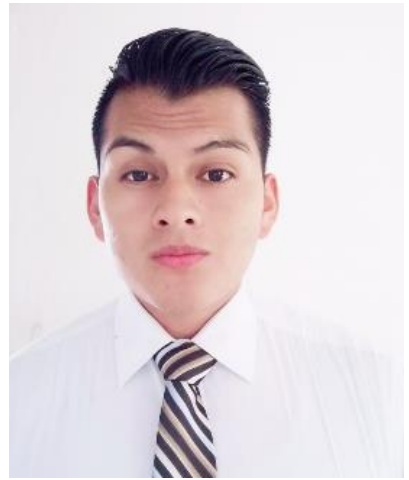

5808-2975.

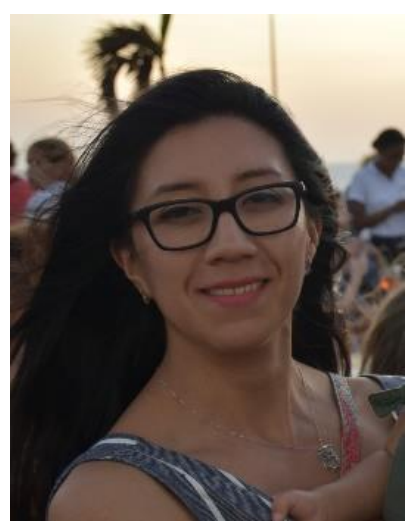

Jonathan Quijia Pillajo, es Ingeniero en Ciencias Económicas y Financieras de la Escuela Politécnica Nacional. Actualmente se desempeña como investigador independiente. Sus áreas de interés son economía industrial, economía de la innovación y economía del bienestar.

Identificador digital ORCID: https://orcid.org/0000-0001-

\section{Carolina Guevara-Rosero, es} Ph.D. en Economía y actualmente es docente del Departamento de Economía Cuantitativa de la Escuela Politécnica Nacional. Sus áreas de interés están relacionadas con la Economía de la Innovación, Economía Regional y Urbana y Economía del Bienestar.

Identificador digital ORCID: https://orcid.org/0000-00017605-1443.

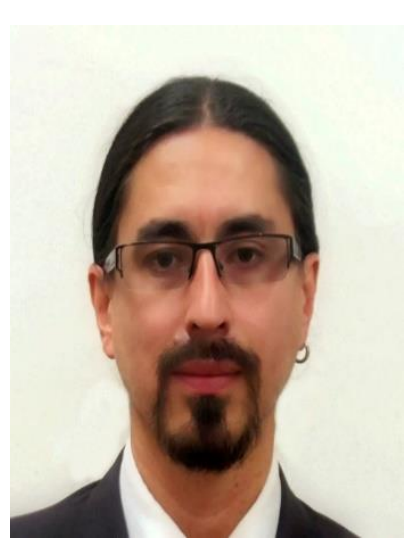

José Ramírez Álvarez. Maestro en Economía del Desarrollo. Doctor en Economía del Desarrollo. Actualmente es Profesor del Departamento de Economía Cuantitativa de la Escuela Politécnica Nacional y en la Facultad de Economía de la Pontificia Universidad Católica del Ecuador. Trabajó como experto tributario en el Servicio de Rentas Internas y analista en el Banco Central de Ecuador. También ha sido consultor del Banco Mundial y el Banco Interamericano de Desarrollo Sus áreas de interés son: Microeconomía, Hacienda Pública, Econometría, Optimización, Programación.

Identificador digital ORCID. https://orcid.org/0000-00019624-3549. 